\title{
13 Schweizerdeutsch und Sprachbewusstsein: Konsolidierung der Diglossie
}

\subsection{Interdependenzen von Gesellschafts- und Sprachbewusstseinsgeschichte}

Unter kulturgeschichtlicher Perspektive ist von einem engen Zusammenhang zwischen sprachbewusstseinsgeschichtlichen Prozessen und politik-, sozial-, wirtschafts- sowie ideengeschichtlichen Entwicklungen auszugehen. Die politischen Veränderungen und der beschleunigte ökonomische und technische Fortschritt prägten auch in der Deutschschweiz des 19. Jahrhunderts nicht nur den Sprachgebrauch, sondern schufen auch die Bedingungen für die sprachbewusstseinsgeschichtlichen Entwicklungen wesentlich mit.

Wie in anderen europäischen Staaten war ideologiegeschichtlich der Patriotismus respektive Nationalismus auch in der Schweiz im 19. Jahrhundert politisch epochenbildend. Aus dem aufgeklärten Patriotismus der Schweizer Eliten im ausgehenden 18. und frühen 19. Jahrhundert hervorgegangen, gewann die Nationalstaatenideologie in der ersten Jahrhunderthälfte deutlich an Popularität. In dem Masse wie ,Volk‘ und ,Vaterland“ beziehungsweise ,Nation“ in diesem Prozess als neue politische und kulturelle Bezugsgrössen relevant wurden, wurden sie auch zu Referenzpunkten der zeitgenössischen Sprachreflexion. Die ,nationale، Bedeutung, die im Rahmen dieses Prozesses den Schweizer Mundarten beigemessen wurde, ist vor diesem Hintergrund zu verstehen. Mit der Bundesstaatsgründung von 1848 erhielt die Schweizer Staatsnation politische und strukturelle Faktizität, in deren Folge sich die nationalintegrativen Prozesse zusätzlich verstärkten, so dass der Nationalismus gegen Ende des 19. Jahrhunderts breitenwirksam war. Es weist zudem einiges darauf hin, dass die Bedrohungskulisse der expansiven Politik Bismarcks und die deutsche Nationalstaatengründung der Reflexion über die politische und kulturelle Bedeutung des Schweizerdeutschen ab den 1860er Jahren neue Schubkraft verliehen haben.

Aus sozioökonomischer Perspektive war die deutsche Schweiz des 19. Jahrhunderts durch vielfältige Modernisierungseffekte im Gefolge der Industrialisierung geprägt, die sich ebenso sehr auf den Sprachgebrauch wie auf die zeitgenössischen Ausformungen der Sprachreflexion auswirkten. Wie in dieser Arbeit gezeigt, wurde das gruppenspezifische Selbstverständnis des deutschschweizerischen Bürgertums, das sich damals politisch und ökonomisch emanzipierte, im zweiten Viertel des 19. Jahrhunderts nicht zuletzt über die Frage nach den Möglichkeiten und Grenzen der Dialekte in einer modernen republikanischen Gesellschaft ausgehandelt. Das patriotisch motivierte Bekenntnis der wirt-

Ә Open Access. (c) 2019 Emanuel Ruoss, publiziert von De Gruyter. (cc)BY-NC-ND Dieses Werk ist lizenziert unter der Creative Commons Attribution-NonCommercial-NoDerivatives 4.0 Lizenz. 
schaftlichen, kulturellen und auch politischen Eliten zum Schweizerdeutschen konsolidierte in der Folgezeit die für die deutsche Schweiz noch heute typische Diglossiesituation und das damit verbundene Bewusstsein einer spezifisch deutschschweizerischen Sprachkultur.

Zugleich strukturierte der rasante sozioökonomische Wandel die sprachbewusstseinsgeschichtlichen Entwicklungen dahingehend mit, dass im Laufe des 19. Jahrhunderts neue Kommunikationsbedürfnisse und -erfordernisse geschaffen wurden, die zu einer Verschiebung der Varietätenverteilung zuungunsten der Mundarten führten. Gerade die zunehmende Mobilität sowie die Einführung der obligatorischen Schulpflicht und die damit zusammenhängende flächendeckende Volksliterarität sind mittelfristig als wichtige Faktoren sprachgeschichtlicher Veränderung in der Deutschschweiz zu werten. Sie führten $\mathrm{zu}$ einem binnendialektalen Ausgleich und zur massiven Übernahme hochdeutschen Sprachmaterials in die Dialekte und prägten damit nicht nur den Sprachgebrauch, sondern indirekt auch die Anfänge einer puristischen Sprachkritik und Dialektpflege, die seit den 1860er Jahren den sprachlichen Wandel als Dialektverfall entschieden ablehnte und bekämpfte.

Insgesamt zeigen die Ergebnisse dieser Arbeit, dass die spezifischen politischen, wirtschaftlichen, sozialen und ideologischen Entwicklungen der (Deutsch-)Schweiz im 19. Jahrhundert wichtige Rahmenbedingungen und integrale Bezugspunkte für die zeitgenössische Sprachreflexion und die darin rekonstruierbaren Sprachbewusstseinsinhalte darstellten. Als solche strukturierten sie die sprachbewusstseinsgeschichtlichen Prozesse massgeblich mit und können erklärende Kraft für deren diachrone Kontinuität und Variabilität haben. Umgekehrt wird in den Analysen aber auch deutlich, dass Sprache als Gegenstand öffentlicher Reflexion ebenso wie die symbolische Besetzung von Varietäten und deren Gebrauch ihrerseits auf ideologische und gesellschaftliche Prozesse zurückwirken. Im Deutschschweizer Kontext des 19. Jahrhunderts betrifft dies in erster Linie die nationale Selbstverständigung, die bis heute auch über die Bezugnahme auf eine eigene Deutschschweizer Sprachgemeinschaft geführt wird. Aber auch auf spezifische Entwicklungen im Schulwesen, insbesondere in der Sprachdidaktik und im Sprachunterricht, haben die rekonstruierbaren Sprachauffassungen ganz wesentlich eingewirkt, wie die Ergebnisse dieser Arbeit zeigen.

Der empirisch eindeutige Nachweis solcher Interdependenzen bleibt jedoch letztlich immer schwierig, weil es sich dabei nicht um einfache UrsacheWirkungs-Verhältnisse handelt. Entsprechende Thesen zum Zusammenhang von Gesellschaftsgeschichte und Sprachbewusstseinsgeschichte können deshalb prinzipiell nur hypothetischen Charakter beanspruchen und ihr Wert muss an ihrer Plausibilität und der Stichhaltigkeit ihrer empirischen Evidenz gemes- 
sen werden. Dabei dürfen sie jedoch solange historische Erklärungskraft beanspruchen, bis sie durch künftige Forschung widerlegt oder differenziert sind.

\subsection{Ausbildung eines nationalen Sprachbewusstseins auf Grundlage der Dialekte}

$\mathrm{Zu}$ den zentralen sprachbewusstseinsgeschichtlichen Prozessen in der Deutschschweiz des 19. Jahrhunderts zählt die Herausbildung eines nationalen Sprachbewusstseins auf Basis der Dialekte bzw. des Schweizerdeutschen. Im Grundsatz wurde diese These bereits formuliert. ${ }^{1}$ Der Zugewinn der vorliegenden Arbeit liegt nun einerseits darin, diese Vermutung empirisch breit fundiert und bestätigt $\mathrm{zu}$ haben; andererseits wurden neue empirische Erkenntnisse zu zentralen Prinzipien herausgearbeitet, die sich als konstitutiv für diesen sprachbewusstseinsgeschichtlichen Prozess erweisen und im Folgenden dargelegt werden.

(1) Eigensprachlichkeitsbewusstsein: Bei der Ausbildung eines nationalen Sprachbewusstseins auf Grundlage der Dialekte spielt zunächst die Konsolidierung eines Eigensprachlichkeitsbewusstseins der Dialekte, also die Überzeugung, dass es sich um historische Varietäten bzw. ,Sprachen“ eigenen Rechts handelt, eine zentrale Rolle. Konstitutiv für diese Entwicklung ist die Überwindung der populären Auffassung, bei den Mundarten handle es sich um ,korrupte' Formen des Hochdeutschen. Diese Vorstellung findet in den ersten Jahrzehnten noch eine gewisse Verbreitung, nimmt im Laufe des Jahrhunderts aber schrittweise ab und wirkt bis am Ende des 19. Jahrhunderts nur noch in Einzelfällen nach. Als wichtige Voraussetzung für diesen paradigmatischen Wechsel der Sicht auf die Dialekte darf deren historische Perspektivierung durch die historische Sprachwissenschaft im Gefolge von Jacob Grimm gelten. Ebenso trägt die im Entstehen begriffene Dialektologie, welche ihrerseits die Eigensprachlichkeit der diatopischen Varietäten betont, vor allem durch mundartgrammatische Arbeiten dazu bei, dass die Mundarten nicht nur als historisch legitimierte, sondern auch als sprachstrukturell regelhafte Sprachformen wahrgenommen werden. Dieses neue Dialektverständnis dominiert bereits im zweiten Viertel des Jahrhunderts den öffentlichen Diskurs in der deutschen Schweiz, indem nun topisch das hohe Alter, der besondere lexikalische Reichtum und darüber hinaus die sprachstrukturelle Regelhaftigkeit der Dialekte hervorgehoben werden. Manifest wird die Vorstellung sprachlicher Autonomie der Dialekte in der

1 Vgl. Sonderegger 2003: 2860. 
Folge vor allem darin, dass ihnen - sie ontologisierend und anthropomorphisierend - ein eigenes ,Wesen` bzw. ein eigener ,Charakter` zugeschrieben wird.

(2) ,Erfindung“ des Schweizerdeutschen: Parallel dazu trägt die ,Erfindung“ des Schweizerdeutschen als nationale Varietät zur Behauptung einer gegenüber dem Hochdeutschen eigenständigen Schweizer Sprache bei - eine „Homogenitätsfiktion“,2 die sich begrifflich in Bezeichnungen wie ,Schweizerdeutsch“, ,Schweizer Mundart‘, ,Schweizerdialekt‘, ,Schweizersprache“ oder ,Nationalsprache“ spiegelt. Über die sprachliche Selbstverortung in der lokalen Sprechergemeinschaft hinaus verstand man sich nun vor allem auch als Teil einer auf dem Schweizerdeutschen basierenden nationalen Sprachgemeinschaft. Schweizerdeutsch steht fortan nicht selten als ,eigentliche“ Muttersprache der Deutschschweizerinnen und -schweizer dem Hochdeutschen als eine Art Fremdsprache gegenüber. Diese Fremdsprachenideologie erweist sich insbesondere auch in den didaktischen Überlegungen zum Hochdeutscherwerb in der Schule als produktiv. Überhaupt sieht man es als pädagogisch wichtige Aufgabe der Schule, das Bewusstsein dialektaler Eigensprachlichkeit an die heranwachsenden Generationen weiterzugeben.

(3) Sprachloyalität: Ein weiterer Faktor, der zur Ausbildung des Schweizerdeutschbewusstseins beiträgt, ist ein hohes Mass an Sprachloyalität, welches die deutschsprachigen Schweizerinnen und Schweizer im 19. Jahrhundert auszeichnet. Sie äussert sich nicht nur darin, dass ein Grossteil des gebildeten Bürgertums an seinen lokalen Dialekten als Alltagsvarietäten festhält, sondern auch darin, dass Versuche von Autochthonen, im Alltag hochdeutsch zu reden, als moralisch fragwürdig und unschweizerisch abgelehnt werden und sich nicht in breiteren Kreisen durchsetzen können. Dabei leuchtet ein, dass auch die Gefahr, mit dem Dialekt einen zentralen Marker der eigenen, (deutsch-)schweizerischen Identität zu verlieren, ex negativo die Loyalität des Bürgertums gegenüber dem Dialekt mitbewirkte und festigte. Dass im Bürgertum der Dialekt beibehalten wird, ist in der historischen Rückschau für die mittel- und längerfristige Konsolidierung des Diglossiebewusstseins sowie den damit verbundenen Erhalt der Diglossiesituation in der Deutschschweiz entscheidend. Aber auch der Bezug zu Vaterland und Nation wirkt sich in Form eines sprachlichen Patriotismus positiv auf die Einstellung gegenüber dem Schweizerdeutschen und dessen Akzeptanz als Alltagsvarietät aller Schichten aus.

(4) Dialektideologie: Ebenfalls nachhaltig beeinflusst eine ausgeprägte Dialektideologie die Ausbildung eines nationalen Sprachbewusstseins. Unter ,Dialektideologie، (ideology of dialect) verstehe ich mit Richard Watts, dessen Definition ich - für das 19. Jahrhundert leicht modifiziert - übernehme:

2 Stukenbrock 2005b: 432. 
any set of beliefs about language in which, in a scenario in which a standardized written language coexists with a number of non-standard oral dialect varieties, the symbolic value of the dialects in some of the linguistic marketplaces in which they are in competition with the standard is not only believed to be much higher than that of the standard but is also deliberately promoted as having a higher value. ${ }^{3}$

Eine so verstandene Dialektideologie bildet sich im Laufe des 19. Jahrhunderts zur zentralen Sprachideologie in der deutschsprachigen Schweiz aus. Sie ist letztlich grundlegend für die verbreitete Überzeugung, die deutschschweizerischen Dialekte bzw. das Schweizerdeutsche weise aus sprachlicher und kommunikativer Sicht eine besondere Wertigkeit und Relevanz auf, die im Falle eines Verlusts nicht einfach durch die Standardsprache zu kompensieren wären.

Dieser deutschschweizerische Dialektismus ${ }^{4}$ des 19. Jahrhunderts kann als Spielart des Vernakularismus verstanden werden, also der Überzeugung, „dass die autochthonen, die lokale/regionale Identität tragenden Sprachen/Varietäten besser/förderungswürdiger sind als Sprachen/Varietäten/sprachliche Formen mit größerer Reichweite“. ${ }^{5}$ Im europäischen Kontext darf in dieser Ideologie eine wichtige Antriebskraft für die Emanzipation der Volkssprachen gegenüber dem Latein und deren Ausbildung zu National- und Kultursprachen gesehen werden. ${ }^{6}$ Diesen Prozess der Vernakularisierung (vernacularization) definiert bereits früh Cobarrubias als „the restoration and/or elaboration of an indigenous language and its adoption as an official language". ${ }^{7}$ Sowohl die historische Ausbildung der neuhochdeutschen Standardsprache als auch die jüngst erfolgte Standardisierung des Lëtzebuergesch und dessen Ernennung zur luxemburgischen Nationalsprache dürfen in diesem Sinne als Vernakularisierungsprozesse verstanden werden.

3 Watts 1999: 69, Kursives ergänzt durch E. R. Bei Watts, der damit die deutschschweizerische Sprachideologie in der zweiten Hälfte des 20. Jahrhunderts beschreibt, heisst es anstelle des von mir kursiv ergänzten ,in some of the linguistic marketplaces“ „in the majority of the linguistic marketplaces“. Damit trägt er der sehr starken Position der Dialekte am Ende des 20. Jahrhunderts Rechnung. Für das 19. Jahrhundert, in dem das Hochdeutsche aufgrund seiner neuen gesellschaftlichen Bedeutung an Domänen dazugewann, musste diese Formulierung entsprechend abgeschwächt werden.

4 Analog zur Begriffsbildung bei anderen Sprachideologien (z. B. ,Konservatismus‘, ,Standardismus', ,Purismus') soll für die Dialektideologie an dieser Stelle der Begriff ,Dialektismus vorgeschlagen werden. Der Begriff scheint terminologisch unproblematisch, auch wenn er in der Sprachwissenschaft bereits die Bedeutung ,dialektaler Ausdruck trägt, wobei er in dieser Bedeutung aber vornehmlich im Plural (,Dialektismen') verwendet wird, während es sich in Bezug auf die Sprachideologie gerade umgekehrt verhält.

5 Maitz/Elspaß 2013: 40.

6 Vgl. Haarmann 1988: 46-49, 1999.

7 Cobarrubias 1983: 66. 
Im Unterschied zu diesen Beispielen des europäischen Vernakularismus, die auf eine Emanzipation der Volkssprache bzw. eines Dialektes zu einer standardisierten National- und Kultursprache zielen, geht es in der deutschen Schweiz des 19. Jahrhunderts vor dem Hintergrund einer bereits etablierten medialen Diglossie mit endoglossischem Standard viel eher darum, die Stellung der Substandardvarietäten innerhalb des bestehenden Varietätengefüges zu behaupten. Die Dialekte werden in ihrem Wert zwar erhöht, nicht aber verabsolutiert. Die Überzeugung einer besonderen Qualität und Güte der Dialekte sowie eine damit zusammenhängende Überlegenheitsbehauptung bleibt auf gewisse, insbesondere nähesprachliche Domänen der Mündlichkeit beschränkt, während in stärker formalisierten Redesituationen sowie im Hinblick auf die Schrift den Vernakularsprachen im Vergleich mit der Standardvarietät keine höhere Wertigkeit zugeschrieben wird.

(5) Sprachpatriotismus: Die dem Dialektismus bzw. der Dialektideologie inhärente Auffassung, das Schweizerdeutsche sei in gewissen Kommunikationszusammenhängen besser geeignet als die überregionale Standardvarietät, ist sprachideologiegeschichtlich nicht $\mathrm{zu}$ trennen von einem deutschschweizerischen Sprachpatriotismus. Als allgemeine Kennzeichen des Sprachpatriotismus gelten mit Andreas Gardt erstens das emphatische Sprachlob und zweitens die oft assoziative und argumentativ wenig schlüssige Übereinanderblendung von Sprache mit den kulturell-ethnischen bzw. politischen Grössen ,Volk`,Nation“ und die daraus resultierende Identifikation von ,Sprach-“ und ,Volks-“ bzw. ,Nationalcharakter ${ }^{8}{ }^{8}$ Wie die Analysen der Schweizerdeutschdiskurse gezeigt haben, lässt sich auf Grundlage dieser Charakteristika auch in der deutschen Schweiz von einer sprachpatriotischen Grundhaltung sprechen. Sie äussert sich im diskursiven Nachweis der Wertigkeit der Dialekte sowie in der Korrelation der Dialekte bzw. des Schweizerdeutschen mit der Schweiz als historisch-ethnischer, kultureller und politisch-territorialer Bezugsgrösse.

Diese sprachpatriotische Haltung, die im zweiten Viertel des Jahrhunderts bereits zu beobachten ist und sich in der zweiten Jahrhunderthälfte akzentuiert, ist vor dem Hintergrund des schweizerischen Nationalismus des 19. Jahrhunderts $\mathrm{zu}$ sehen. Im Kontext des schweizerischen Nation-Building spielt die Besinnung auf eine gemeinsame Vergangenheit und gemeinsam geteilte Kulturgüter eine entscheidende Rolle. Die Verständigung über das sprachliche Erbe dient dabei der binnen(deutsch)schweizerischen Integration und der Konstruktion

8 Vgl. Gardt 1999a: 302, 1999b: 92, 2000d: 248; ferner auch die ,Prinzipien“ des Sprachnationalismus bei Stukenbrock 2005b: 431-442, von denen das ,Prinzip der Heterogenitätsreduktion‘, das ,Sprach- und Selbstlob،, das ,Korrelationsprinzip“ oder das ,deontische Prinzip“ auch auf den Deutschschweizer Sprachpatriotismus des 19. Jahrhunderts zutreffen. 
einer schweizerdeutschen Sprachgemeinschaft. Spätestens in der zweiten Jahrhunderthälfte gewinnt ergänzend auch die nationalsymbolische Abgrenzungsfunktion des Schweizerdeutschen an Bedeutung. Mit der durch den Bürgerkrieg erzwungenen Bundesstaatsgründung von 1848 werden die Glieder der Eidgenossenschaft zu einem mehrsprachigen Nationalstaat. Die nationale Zugehörigkeit und politische Integrität der deutschen Schweiz stehen spätestens zu diesem Zeitpunkt ausser Frage. Vor dem Hintergrund des deutschen Sprachnationalismus und der Entstehung des Deutschen Reichs 1871 wird entsprechend ausdrücklich das sprachlich Trennende betont und das Schweizerdeutsche zu einem Symbol nicht nur der sprachlichen, sondern auch der politischen Abgrenzung gegenüber Deutschland stilisiert. Das Bewusstsein einer eigenen Sprache wird so im 19. Jahrhundert insgesamt zu einem wichtigen Moment kollektiver Identität in der deutschsprachigen Schweiz, das in dem Masse auch ein Moment ,nationaler Identitätsstiftung ist, wie es politische Abgrenzungsfunktion gegen die deutschsprachigen Nachbarstaaten übernimmt.

Es ist jedoch nachgerade charakteristisch für die Sprachbewusstseinsgeschichte der deutschen Schweiz im 19. Jahrhundert, dass es zwar einen Sprachpatriotismus gibt, der seinen Teil zur Wertschätzung der Dialekte beiträgt, dass er sich aber nie zu einem eigentlichen Sprachnationalismus entwickelt. ${ }^{9}$ Zwar wird auch in der deutschen Schweiz geltend gemacht, dass durch den Sprachkontakt mit dem Hochdeutschen und die damit einhergehende ,Verunreinigung des Dialekts die sprachliche und kulturelle (deutsch-)schweizerische Identität gefährdet sei. Um von einem Sprachnationalismus sprechen zu können, fehlt jedoch eine chauvinistische Ausrichtung, welche die absolute Überlegenheit des Schweizerdeutschen gegenüber dem Hochdeutschen behaupten und das Hochdeutsche als Fremdes per se desavouieren und ablehnen würde. Stattdessen wird der Vorrang der Dialekte nur partiell und nur in Bezug auf die Mündlichkeit behauptet, während generell der kulturelle und kommunikative Wert der Standardsprache zu keinem Zeitpunkt infrage gestellt wird. Damit unterscheidet sich der schweizerische Sprachpatriotismus auch von einem in Deutschland dominierenden kulturchauvinistischen Sprachnationalismus, der sich seit dem 19. Jahrhundert vor allem gegen alles Französische richtet. ${ }^{10}$

$9 \mathrm{Zu}$ dieser typologischen Unterscheidung vgl. Gardt 1999a: 302, 1999b: 91-92, 2000d: 247248. Nach Gardt teilen die beiden Konzepte die Merkmale (1) des Sprachlobs und (2) der Übereinanderblendung von Sprache mit ,Volk`/,Nation'. Für den Sprachnationalismus kommen als weitere Kriterien (3) der (sprachliche) Überlegenheitsanspruch sowie (4) die Behauptung der Gefährdung der eigenen Sprach- und Volksgemeinschaft durch fremden Einfluss hinzu (vgl. Gardt 1999b: 92; 107-109).

10 Vgl. Gardt 2000d. Vor dem Hintergrund der Zuspitzung der deutsch-französischen Erbfeindschaft stilisierten um die Wende zum 20. Jahrhundert aber auch Mitglieder germanophiler 
Ein wichtiger Grund dafür, dass sich in der deutschen Schweiz kein Sprachnationalismus ausbildet, ist die im Vergleich zu Deutschland sehr unterschiedliche historische und soziolinguistische Ausgangssituation. Die Schweiz ist seit Jahrhunderten territorial mehrsprachig. Ihr nationales Selbstverständnis gründet nicht auf einer quasi-natürlichen sprachlich-kulturellen, sondern auf einer politischen Zusammengehörigkeit, weshalb auch das Modell einer Sprachnation keine Option darstellt. Das Ausbleiben eines chauvinistischen Sprachnationalismus ist überdies dem Spannungsfeld einer doppelten, das heisst politischkulturellen und sprachlich-kulturellen Selbstverortung der verschiedenen Landesteile geschuldet. So, wie man sich politisch als schweizerisch begreift, versteht man sich sprachlich und kulturell zugleich als Teil einer grösseren Gemeinschaft, deren Hauptzentren die sich ausbildenden grossen Nachbarstaaten Frankreich, Italien und Deutschland bilden. Unter diesen Voraussetzungen ist es für die deutsche Schweiz weder opportun sich sprachlich vom deutschen Kulturraum abzuspalten, noch sich als deutsche Schweiz politisch der deutschen ,Sprachnation' anzuschliessen.

In dem Masse, wie der Sprachpatriotismus die Wertigkeit des Dialekts bzw. des Schweizerdeutschen zusätzlich noch steigert, ist er auch an der Festigung der oben erwähnten Dialektideologie beteiligt. Dialektismus und Sprachpatriotismus sind seither in der deutschen Schweiz unauflöslich ineinander verzahnt. Dass der sprachliche Patriotismus auf den Dialekten und nicht auf der überregionalen Schriftsprache basiert, unterscheidet ihn von den Entwicklungen in Deutschland und in anderen europäischen Ländern. ${ }^{11}$

Es ist also, alles in allem, ein Zusammenspiel vielfältiger Faktoren, die im Laufe des 19. Jahrhunderts ein spezifisch schweizerisches Sprachbewusstsein auf Grundlage der Dialekte bzw. des Schweizerdeutschen ausgebildet und die diatopischen Varietäten in der Schweiz als wichtiges kulturelles Erbe und als Merkmal schweizerischer Nationalität konstituiert haben.

und pangermanischer Kreise der Schweiz das (sprachliche) Verhältnis zwischen Deutsch und Französisch zu einer sprachlich-kulturellen Existenzfrage (vgl. Müller 1977). Dieser sogenannte Sprachenstreit darf als Symptom jener sich zuspitzenden binnenschweizerischen Konfliktlinien entlang der Sprachgrenzen gelten, die mit dem Ausbruch des Ersten Weltkrieges die Schweiz vor eine bis dahin einmalige innenpolitische Zerreissprobe stellten (vgl. Müller 1977: 116; Maissen 2010b: 241-242; Tanner 2015: 128-131).

$11 \mathrm{Zu}$ Deutschland vgl. Gardt 2000d; Stukenbrock 2005b; zur Bedeutung der Einheitssprache im Kontext der europäischen Nationalismen vgl. Blommaert/Verschueren 1998. 


\subsection{Diglossie als Konstituens der deutschschweizerischen Sprachkultur}

Neben der Ausbildung eines nationalen Sprachbewusstseins auf Grundlage des Schweizerdeutschen zählt die Konsolidierung eines kollektiven Diglossiebewusstseins $\mathrm{zu}$ den zentralen sprachbewusstseinsgeschichtlichen Prozessen in der deutschen Schweiz des 19. Jahrhunderts. Die Auseinandersetzung mit der Diglossie, die in der Variante mit totaler Überlagerung definitiv zu einem soziolinguistischen ,Sonderfall' wird, wird zum Kristallisationspunkt der Schweizerdeutschdiskurse dieser Zeit.

Die Legitimierung der Dialekte als Alltagsvarietäten und die Aushandlung der konkreten pragmanormativen Bedingungen des Varietätenmodells beherrschen als vordringlichste sprachreflexive Themen die erste Jahrhunderthälfte. Entscheidend dabei ist, dass Dialekt und Hochdeutsch als kategorial verschiedene ,Wesen' konzeptualisiert werden, die sich mit ihren je eigenen Vorzügen in der Kommunikation funktional wechselseitig ergänzen. Diese kommunikationsfunktionale Komplementarität der beiden Varietäten wird in der Folge ebenso wenig infrage gestellt, wie die daraus abgeleiteten pragmatischen Normvorstellungen im Hinblick auf die Varietätenverteilung. Dialekt und Hochdeutsch gelten fortan als zwei legitime Sprachformen, die jeweils ,an ihrem Ort` ihre Berechtigung beweisen: der Dialekt in der Face-to-face-Interaktion in Nähesituationen, das Hochdeutsche in formalisierten Kommunikationssituationen und in der Schrift. Ergänzt werden diese zunächst vor allem pragmatischen Normvorstellungen im letzten Drittel des Jahrhunderts durch das Postulat einer formalen Separierung der Varietäten. So stabilisieren sich im 19. Jahrhundert schliesslich (später als typisch für Diglossiesituationen beschriebene ${ }^{12}$ ) usuelle Normen wie die klare sprachlich-formale und funktionale Trennung zwischen Dialekt und Hochdeutsch sowie die Verwendung des Dialekts in allen Bevölkerungsschichten.

Charakteristisch für dieses Diglossiebewusstsein ist eine grundsätzliche Wertschätzung beider Varietäten. Neben der besonderen Wertschätzung der Dialekte geniesst auch das Hochdeutsche als Sprache der Schrift sowie der Bildung und Kultur ungebrochen ein hohes Prestige - auch in gesprochener Form. In Vorträgen über wissenschaftlich-kulturelle, aber auch feierlich-erhabene Gegenstände gilt es als die angemessene Ausdrucksweise und je länger je mehr als selbstverständlich. Es ist geradezu kennzeichnend für die deutschschweizerische Sprachkultur des 19. Jahrhunderts, dass die Dialektideologie nicht abso-

12 Vgl. Ferguson 1959. 
lut gilt, sondern mit einer Standardideologie koexistiert, also der Überzeugung, die Standardvarietät einer Sprache sei unerlässliches Mittel der Bildung und Bedingung des gesellschaftlichen Fortschritts und in dieser Hinsicht die bedeutsamste Varietät dieser Sprache. ${ }^{13}$ Wie in Deutschland manifestiert sich dieser Standardismus im 19. Jahrhundert auch in der Deutschschweiz zunächst vor allem als Homogenitätsideologie, der zufolge sprachliche Variation in der Standardsprache negativ ist. Erst allmählich wird diese Auffassung aufgeweicht und auch der Gebrauch bestimmter helvetischer Besonderheiten in Aussprache und Lexik legitimiert.

Auch ist nicht nur gegenüber den Mundarten, sondern auch gegenüber der Standardvarietät von einer gewissen Sprachloyalität auszugehen. Diese speist sich aus dem Selbstverständnis, dass die neuhochdeutsche Schriftsprache nicht von aussen aufoktroyiert ist, sondern sich historisch unter kontinuierlicher deutschschweizerischer Mitwirkung herausgebildet hat. Stärker freilich als in Bezug auf seine mündliche Realisierung wird die neuhochdeutsche Schriftsprache als eigene, zur Deutschschweiz gehörige Schreibvarietät verstanden. Von diesem Selbstverständnis zeugen die damals wie heute gebräuchlichen Begriffe ,Schriftsprache“ und ,Schriftdeutsch'. ${ }^{14}$ Auf Grundlage der Deutschschweizer Dialekte eine einheitliche Schrift- und Nationalsprache $\mathrm{zu}$ schaffen, war deshalb keine Option. Eine ,Hollandisierung‘, also eine sprachliche Abspaltung vom deutschen Kulturraum, wollte man weder aus kulturellen noch aus wirtschaftlichen Gründen riskieren.

Die funktionale Verteilung der Varietäten in der gesprochenen Sprache und der fast ausschliessliche Gebrauch des Hochdeutschen in der Schrift werden im Laufe des Jahrhunderts zu einer zentralen Konstituente deutschschweizerischer Sprachkultur. Sprachbewusstseinsgeschichtlich zeichnet sich das 19. Jahrhundert nicht durch die Überzeugung aus, die Volkssprache müsse sich zur schweizerischen National- und Kultursprache emanzipieren und die deutsche Standardvarietät als ,fremde“ Sprache verdrängen. Vielmehr konsolidiert sich die Auffassung, den lokalen Dialekten stehe im Bereich der gesprochenen Sprache ihr historisches, dem Schweizerdeutschen sein nationales Recht $\mathrm{zu}$, während das Hochdeutsche als Kultur- und Gemeinsprache die Dialekte weiterhin überdachen solle. Alternativ zur gesellschaftlichen Monoglossie und zum Streben nach einer einheitlichen Nationalsprache in Deutschland verkörpert die Diglossie

13 Vgl. Milroy/Milroy 1999 [1985]; Milroy 2001; Maitz/Elspaß 2013: 35.

14 Die Begriffe können bis heute sowohl geschriebenen als auch gesprochenen Standard bezeichnen und verweisen damit noch einmal auf die Wahrnehmung und Funktion der Standardvarietät als beinahe exklusive Varietät des Schriftlichen und ihre lange Zeit marginale Bedeutung für die gesprochene Sprache. 
zunehmend ein konstitutives Merkmal nationaler Sprachkultur in der (deutschsprachigen) Schweiz. Der diglossische ,Sonderweg' wurde so neben dem nationalen Sprachbewusstsein auf Basis des Schweizerdeutschen als QuasiNationalsprache $\mathrm{zu}$ einem weiteren zentralen Moment deutschschweizerischnationaler Identifikation.

Um Missverständnissen vorzubeugen, ist nach dem Gesagten noch Folgendes anzufügen: Wenn von einer Konsolidierung und mithin einer Stabilisierung des Diglossiebewusstseins im 19. Jahrhundert die Rede ist, bedeutet das nicht, dass die Diglossiesituation als solche stabil gewesen wäre. Diglossiesituationen sind prinzipiell instabil, insofern sich die Verteilung der Varietäten diachron fortwährend verschiebt und sich innerhalb einer diglossischen Sprachgemeinschaft die Aushandlung ihrer Funktionen und Domänen als kontinuierlicher Prozess darstellt. Dies belegen die sprachgebrauchsgeschichtlichen Entwicklungen in der Deutschschweiz des 19. und 20. Jahrhunderts ebenso wie die damit eng verknüpften Diglossiediskurse. Während sich die Varietätenverteilung im 19. Jahrhundert deutlich zugunsten des Hochdeutschen verschob, ist im 20. Jahrhundert spätestens seit den 1930er Jahren eine Gegenbewegung im Gange, durch die die Dialekte teils in Domänen, die zwischenzeitlich hochdeutsch waren, teils in neuen Kommunikationssituationen gebräuchlich wurden. Ungeachtet solcher diachronen Verschiebungsprozesse im Varietätengefüge blieben letztlich das grundsätzliche diglossische Selbstverständnis und die damit zusammenhängenden basalen Kommunikationsmaximen in der Deutschschweiz seit Mitte des 19. Jahrhunderts erhalten.

\subsection{Sonderfall Schweiz?}

Im 19. Jahrhundert schlug die (deutsche) Schweiz nicht nur politisch und wirtschaftlich, sondern auch sprachgeschichtlich den „Weg zur Gegenwart“15 ein. Mit Blick auf die Diglossiesituation wird die deutschsprachige Schweiz seither von aussen, aber gerade auch von innen immer wieder als sprachlicher Sonderfall wahrgenommen. Dieses Sonderfalldenken hat in der (deutsch) Schweizerischen Selbstwahrnehmung eine gewisse Tradition und spielt nicht nur mit Blick auf die Sprache seit dem ausgehenden 19. Jahrhundert eine Rolle. ${ }^{16}$

15 So der Titel der Überblicksdarstellung zur Schweiz im 19. Jahrhundert von Kreis 1986.

16 Die Rede vom ,Sonderfall Schweiz‘ geht davon aus, dass der Schweiz aufgrund ihrer Geschichte, ihrer geographischen Lage, ihres politischen Systems und ihrer Kultur eine Sonderstellung und teilweise auch eine Vorbildsfunktion innerhalb der Staatengemeinschaft zukomme. Während das Sonderfalldenken lange positiv besetzt war, geriet es in gesellschaftskritischen Kreisen seit den 1970er Jahren zunehmend in Kritik; seit den 1990er Jahren wird das 
Inwiefern ging die Schweiz nun aber mit ihrer sprachbewusstseinsgeschichtlichen Entwicklung einen Sonderweg? Mit der diglossischen Varietätenverteilung nimmt die Schweiz im deutschsprachigen Raum heute soziolinguistisch tatsächlich eine ganz eigene Stellung ein. Die Weichen für diese Entwicklung wurden sprachgebrauchsgeschichtlich bereits im 18. und sprachbewusstseinsgeschichtlich vor allem im 19. Jahrhundert gestellt. Es ist jedoch wichtig zu sehen, dass aus sprachbewusstseinsgeschichtlicher Perspektive die Entwicklungen in der deutschen Schweiz nicht unabhängig von gesamtdeutschsprachigen, teilweise auch von gesamtwesteuropäischen Entwicklungstendenzen stattfanden. In Bezug auf verschiedene Phänomene müsste daher eher von einer schweizerischen Spielart allgemeiner Entwicklungen gesprochen werden als von einem Sonderweg. So lässt sich ab dem ausgehenden 18. Jahrhundert innerhalb des gesamten deutschen Sprachraums ein neues Regionalsprachenbewusstsein feststellen, das im 19. Jahrhundert zu einer grundsätzlichen, wenngleich regional recht unterschiedlichen Aufwertung lokaler und regionaler Sprachformen, insbesondere der Dialekte führt und die Einsicht in deren Eigensprachlichkeit befördert. ${ }^{17}$ Die schweizerische Spielart eines umfassenderen Phänomens manifestiert sich auch im damaligen Sprachpatriotismus auf Grundlage der Dialekte bzw. des Schweizerdeutschen. Zeitlich und thematisch entwickelt er sich spezifisch schweizerisch, strukturell lässt er sich jedoch in eine längere Tradition des deutschen (und europäischen) Sprachpatriotismus einordnen. ${ }^{18}$ Auch die Rückbesinnung auf das sprachlich Eigene als Ausdruck regionaler Identität und die damit einhergehenden Ansätze zur Sprachpflege und Sprachpolitik, die sich in der zweiten Jahrhunderthälfte vor dem Hintergrund einer Modernisierungs- und Kulturkritik abzeichnen, sind keine rein schweizerischen Phänomene, wie etwa Bestrebungen zur Reliterarisierung und Pflege des Niederdeutschen belegen. Dennoch: Aufgrund politisch, gesellschaftlich und sprachlich sehr unterschiedlicher Voraussetzungen hat die deutschschweizerische Sprachbewusstseinsgeschichte des 19. und 20. Jahrhunderts bei allen Interdependenzen ihre eigene, sehr spezifische Ausprägung als veritabler Sonderfall innerhalb der Sprachbewusstseinsgeschichte des Deutschen.

Bild des Sonderfalls von nationalkonservativen Kreisen wieder vermehrt bedient (vgl. Kreis 2012).

17 Vgl. Mattheier 2005.

18 Zum historischen Zusammenhang von Sprache und Nation im deutschsprachigen Raum sowie im Hinblick auf weitere europäische Kultursprachen vgl. die Beiträge in Gardt 2000a. 